\title{
Increasing opportunity through interdisciplinary research: climbing down and shattering a tower of babel
}

\author{
Jacqueline C. Resnick* \\ The Office of Research Development and adjunct faculty, Department of Maternal and Child Health, The School of Public Health at The University of North Carolina, \\ Chapel Hill, NC, USA \\ ${ }^{*}$ Correspondence: jacki_resnick@unc.edu
}

In today's complex funding world, researchers, especially biomedical researchers, are looking toward solving societal problems using powerful new technologies. Exploring problems and questions not confined to a single discipline has set the environment to support collaborative approaches leading to interdisciplinary solutions (Drake and Donohue, 1996). Multidisciplinary teams have always provided unique opportunities for researchers across disciplines to communicate with one another (Gray, 1989). Interdisciplinary approaches set the stage for innovation by uniting together to create new tools, develop new disciplines and ultimately open new avenues of research.

While multidisciplinary research brings disciplines together, interdisciplinary research cuts across the disciplines and fosters the integration of ideas. Lee et al. (2009) paper, look at incorporating collaborator concepts into informatics in support of translational interdisciplinary biomedical research. They discuss the complexities of taking this approach to doing research and look into the environmental factors needing to be in place to support complex collaborations (i.e., data integration and analysis, data sharing, management, and security. In addition, they discuss the need for appropriate training and long-term planning as necessary factors to achieving success. Increasing support at the institutional level begins the process of shattering the tower of babble.

As interdisciplinary projects gain recognition and visibility through publications in major journals (e.g., Science, Nature) because they often offer novel approaches to traditional problems, federal agencies have increased funding opportunities for interdisciplinary research through the creation of centers of excellence and the encouragement of building consortia in anticipation for translational research (Finholt and Olson, 1997). The National Institutes of
Health Clinical Translational Science Award (CTSA) initiative has increased funding opportunities in support of translational research efforts.

Although there is increased interest and support for interdisciplinarity to occur, it is not without problems. As Lee et al. (2009) suggest, scientists are often unwilling to share and trust each other in today's highly competitive funding world. To help deal with these types of issues, the University of North Carolina at Chapel Hill has established a formal publication and data sharing policy in support of eliminating barriers often associated with collaborative efforts. An unwillingness to adapt research tools that are unfamiliar adds to the difficulty of utilizing necessary core technologies (Hara et al., 2003). Team members are frequently hampered by distance and unless a strong infrastructure is in place there is often a day to day communication disconnect allowing for disparate systems to dictate workflow rather than having a synergistic approach (Gibbons et al., 1994).

To assure the success of interdisciplinary/ translational research requires agencies and foundations to provide a strong funding base making it possible for a research team to tackle such problems (Jeffery, 2003). In addition, training opportunities must be provided by the large federal agencies such as The National Institutes of Health Roadmap (NIH; www.roadmap.nih.gov) and The National Science Foundation (NSF) to increase institutional understanding and to ensure output of experts trained in appropriate methodologies.

Bracken and Oughton's (2009) paper on interdisciplinary research made a case for framing and reframing questions and approaches. They highlighted the changing climate on research funding as an impetus for the expansion of interdisciplinary approaches. They provided data from 12 semi-structured interviews with leaders of interdisciplinary research projects that crossed the natural and social sciences. These interviews all occurred within the United Kingdom. Their purpose was to explore the ways in which researchers with different disciplinary backgrounds approached the framing of interdisciplinary questions. They pointed out that "the differences in research cultures and established habits implied very different starting points for the initiation of a research idea" making the case for framing as a tool to facilitate these differences from becoming problematic. This paper built upon their 2006 research and concluded successful projects were able to maintain strong communication over the life time of a project and that self-awareness, and a willingness to be questioned by others was essential components for team building. They emphasized the need to relate different epistemologies and methods not president in disciplinary focused projects to support an expanded approach to understanding how people work well together if research teams are to be strong.

Sa (2008) points out that interdisciplinary strategies in U. S. research universities, emphasized the need for universities to change their current structures by reducing barriers to investigations where research crosses disciplines. He looked at the two recent reports from the National Academy of Sciences (2005) and the Association of American Universities (2005) on how universities can facilitate interdisciplinary research. Innovation calls for new pathways to be opened; engaging scholars with each other so that "leaps in scientific progress can be made and greater economic and social benefits to society" can occur. In his paper he speaks to the ongoing conflict between traditional disciplinary approaches that lead to a continuum of fragmentation of knowledge and the formation of department silos as opposed to centers and research units fostering collaboration. To 
address this type of fragmentation and to help create linkages throughout the campus, the University of North Carolina at Chapel Hill, developed an Office of Research Development in 1996. The purpose of this new program was to establish a pan-university unit that could fill the role of an academic "think tank." The goal of the office was to set an environment for scholars to engage with each other using entrepreneurial "out of the box" approaches in building multi/interdisciplinary research across the campus. The Unit was placed within the Office of the Vice Chancellor for Research and Economic Development to assure it would be seen as being neutral, not associated with any specific college. The Office of Research Development is now in its 17 th year and is viewed as being highly successful in facilitating research development within the University and in building partnerships externally while placing an emphasis on translational research.

The funding of translational projects whose goals include collaborations with communities and industry, having shared resources and equipment, and ultimately leading to the effective dissemination of new knowledge and discovery is important. To achieve this, it is essential that inter-departmental and inter-college collaborations become institutionalized (e.g., educational plans, faculty reward and recognition, and funding support). He points out that as science becomes more expensive, interdisciplinary research is one way to rationalize institutional resources and deal with the ongoing pressure to enhance the academic research enterprise. Establishing a paradigm shift in academia must insure respect for scholars to operate within their own cultures. "One size fits all" is a failing approach to building interdisciplinary research structures. Sa's (2008) paper provides an identification of organizational strategies that foster the interdisciplinary environment while looking at the implications of changes that occurred.

Pless et al. (2010) reported a case study of a consortium model to establish a new field. He pointed out the impact of potentially isolated studies being transformed, through team interactions, into a coherent body of work. He suggested in his case report, team contribution stimulated a previously underdeveloped field of research void of peers or mentors. He also reported that as the new field emerged, the group members were seen as experts helping them to be recognized and promoted within their respective institutions. Outcomes of their work pointed to improved measures, new concepts and interventions and policy. In their case, problems needing to be addressed revolved around group versus individual projects, policy analysis versus intervention research, and issues relating to research and practice. They concluded that the longevity of the consortium and its endurance required that their researchers understood that collaborative thinking integrating research enhances collective accomplishment. Everyone wins! A similar consortium model supported through a program project grant from NIDA to the University of North Carolina, established a multi-disciplinary, multi-institute effort to study cocaine effects on maternal behavior in humans and animal models. This recently funded consortium has led to the establishment of data management and biostatistics core facilities within the partner institutions. These cores help foster multidisciplinary efforts leading to interdisciplinary outcomes through points of convergence and interactions across diverse projects. These models are excellent examples of translational research.

Recommendations from the Pless et al. (2010) paper also included the need for foundations and government agencies to provide support on topics and areas of inquiry not popular or not having sustained funding priority to open doors for creativity and innovation.

Establishing measures of success for interdisciplinary research still needs addressing. Comparative studies pointing to mechanisms providing measures along with studies to better understand campus cultures and factors that influence faculty research behavior are important if positive change is to occur (Zerhouni, 2005). Institutions must take into account that targeted resource allocation often communicates inequity among faculty whose fields are not seen as a priority and find positive ways to bridge this gap. True interdisciplinary research increases opportunity for collaboration providing a place at the table for all disciplines. The 2005 report from the National Academy goes into great detail of finding legitimate and desirable methods to reform campus policies in facilitating interdisciplinarity including studies that compare the experience trajectories of faculty.

Considering widely different norms for evaluation while building consensus, augmenting institutional prestige, and addressing the needs of students to develop the expertise necessary to participate in these endeavors is essential.

Interdisciplinary research is a mode of research by teams or individuals from two or more disciplines or bodies of specialized knowledge that integrate information, data, techniques, tools, perspectives, concepts, and/or theories to advance fundamental understanding or to solve problems whose solutions are beyond the scope of a single discipline (National Academy of Sciences, 2005 report). Translational research is the process of applying ideas, insights, and discoveries generated through basic scientific inquiry. It is the leveraging of these two approaches and the integration and the sharing of data across disciplines that fosters collaborative models. Only with support from funding agencies and the restructuring of higher educational institutions validating faculty involvement can we widen the door for new discoveries thus breaking down the towers of babble.

\section{REFERENCES}

Bracken,L.J., and Oughton,E.A. (2009). Interdisciplinarity within and beyond geography: introduction to special section. Area 41, 371-373.

Drake, L.E., and Donohue, W.A. (1996). Communicative framing theory in conflict resolution. Communic. Res. 23, 297.

Finholt, T.A., and Olson, G. M. (1997). From laboratories to collaboratories: a new organizational form for scientific collaboration. Psychol. Sci. 8, 28-36.

Gibbons, M., Limoges, C., Nowotny, H., Schwartzman, S., Scott, P., and Trow, M. (1994). The New Production of Knowledge: The Dynamics of Science and Research in Contemporary Societies. Thousand Oaks, CA: Sage Publications, Inc.

Gray, B. (1989). Collaborating, Finding Common Ground for Multiparty Problems. San Francisco: Jossey-Bass.

Hara, N., Solomon, P., Kim, S.-L., and Sonnenwald, D. H. (2003). An Emerging view of scientific collaboration: scientists' perspectives on collaboration. J. Am. Soc. Inf. Sci. Technol. 54, 952-965.

Lee, E. S., McDonald, D. W., Anderson, N., and TarczyHornoch, P. (2009). Incorporating collaborator concepts into informatics in support of translational interdisciplinary biomedical research. Int. J. Med. Inform. 78, 10-21. 
Kates, R.W., with National Academy of Sciences Committee on Facilitating Interdisciplinary Research. (2005). Facilitating Interdisciplinary Research. Washington, DC: National Academy Press.

Pless, I. B., Stein, R. K., and Walker, D. (2010). Research consortium on children with chronic conditions (RCCCC): a vehicle for interdisciplinary collaborative research. Matern. Child Health J. 14, 9-19.
Sa, C. M. (2008). Interdisciplinary strategies' in U.S. research universities. High Educ. 55, 537-552.

Zerhouni, E. A. (2005). US biomedical research: basis, translational and clinical sciences. J. Am. Med. Assoc. 294, 11352-11358.

Received: 28 February 2011; accepted: 11 April 2011; published online: 12 May 2011.

Citation: ResnickJC (2011) Increasing opportunity through interdisciplinary research: climbing down and shattering a tower of babel. Front. Psychiatry 2:20. doi: 10.3389/ fpsyt.2011.00020

This article was submitted to Frontiers in Child and Neurodevelopmental Psychiatry, a specialty of Frontiers in Psychiatry.

Copyright $(2011$ Resnick. This is an open-access article subject to a non-exclusive license between the authors and Frontiers Media SA, which permits use, distribution and reproduction in other forums, provided the original authors and source are credited and other Frontiers conditions are complied with. 